\title{
P2: Ein mentalisierungsgestütztes Pädagogik-Konzept in der Ausbildung für Krankenpflegepersonal
}

\author{
Johann Steinberger $\cdot$ Wolfgang Sieberth $\cdot$ Ewa Zeman
}

Online publiziert: 30. Oktober 2013

(C) Springer-Verlag Wien 2013

Einleitung/Fragestellung: Viele Ausbildungen haben die Schwierigkeit, Fähigkeiten, die außerhalb einer kognitiven Erfassung liegen, zu schulen. Seit einigen Jahren belegen Studien die Wichtigkeit einer guten Ausbildung im Bereich von Einfühlung, Gedankenlesen, Theory of Mind usw.

Wie vermittelt man bzw. schult man Fähigkeiten, die zu einer mentalisierenden Haltung führen? Unter Mentalisierung verstehen wir, sich und andere Personen als Wesen mit geistigen und emotionalen Fähigkeiten bzw. mit einer Psyche zu betrachten und reflexiv zu verstehen. Die Fähigkeit zur Mentalisierung entwickelt sich in Abhängigkeit von Bindungs- und Beziehungserfahrungen und deren gemeinsamer Veränderung bzw. Entwicklung.

Methodik: Das Gesamtkonzept dieser Schulung erstreckt sich über sechs Semester. In den beiden ersten Semestern liegt der Schwerpunkt auf der Introspektion, die durch Selbsterfahrung geschult wird. In diesen beiden Semestern geht es um das Wahrnehmen der eigenen Bedürfnisse und Gefühlsreaktionen in diversen Lebenslagen und die damit verknüpften eigenen Lebensnarrative. In den Semestern fünf und sechs geht es schließlich um die Fähigkeit Supervision zu nützen und auch selbstreflexives Verhalten gegenüber den Patienten und Mitarbeitern darzustellen. Der Student sollte nun die Fähigkeit der prozeduralen Verknüpfung von deklarativem Wissen haben.

Wir entwickelten eine Form der „Patientenbeobachtung“ wo es primär um die Schulung und das Nützen einer mentalisierenden inneren Haltung geht. Ziel dieses dreijährigen Projektes waren unter anderem die Schulung von Empathie, Metakognition, innere Achtsamkeit und emotionaler Intelligenz als grundlegende Kompetenz zur Entwicklung reflexiver Handlungskompetenz, die wiederum zu einer Fähigkeit von Mentalisierung zusammengefasst werden kann. Überprüfung: Gießentest II, SPSS

Ergebnisse: Im Zuge unserer Schulung in Sechsergruppen konnten wir eine klare Verbesserung der Mentalisierungsfähigkeit der Studenten feststellen.

J. Steinberger $(\bowtie) \cdot$ W. Sieberth $\cdot$ E. Zeman

Wiener Institut für Pädagogik und Psychotherapie (WIPP),

Wien, Österreich

E-Mail: Johann.steinberger@drei.at 